PROCEEDINGS OF THE

AMERICAN MATHEMATICAL SOCIETY

Volume 137, Number 9, September 2009, Pages 3163-3165

S 0002-9939(09)09915-8

Article electronically published on May 1, 2009

\title{
NON-VANISHING COMPLEX VECTOR FIELDS AND THE EULER CHARACTERISTIC
}

\author{
HOWARD JACOBOWITZ
}

(Communicated by Varghese Mathai)

\begin{abstract}
Every manifold admits a nowhere vanishing complex vector field. If, however, the manifold is compact and orientable and the complex bilinear form associated to a Riemannian metric is never zero when evaluated on the vector field, then the manifold must have zero Euler characteristic.
\end{abstract}

One of the oldest and most basic results in global differential topology relates the topology of a manifold to the zeros of its vector fields. Let $M$ be a compact and orientable manifold and let $\chi(M)$ denote its Euler characteristic. Here is the simplest statement of this relation.

(1) If there is a global nowhere zero vector field on $M$, then $\chi(M)=0$.

This of course is for a real vector field (that is, for a section $M \rightarrow T M$ ). On the other hand, it is easy to see that any manifold admits a nowhere zero complex vector field. (A complex vector field is a section $M \rightarrow \mathbb{C} \otimes T M$.) This can be seen most simply by observing that a generic perturbation of any section, even the zero section itself, must be everywhere different from zero.

It is natural to seek a condition on a nowhere zero complex vector field which would again imply $\chi(M)=0$. Curiously, a trivial restatement of (10) leads to such a condition. Let $g$ be any Riemannian metric on $M$.

(2) Let $v: M \rightarrow T M$ be a global vector field on $M$. If the Riemannian metric $g(v, v)$ is never zero, then $\chi(M)=0$.

Here is the condition for complex vector fields.

Theorem 1. Let $v: M \rightarrow \mathbb{C} \otimes T M$ be a global vector field on $M$. If the bilinear form $g(v, v)$ is never zero, then $\chi(M)=0$.

Here $g$ is extended to complex vector fields by taking $g(v, w)$ to be complex linear in each argument; for $v=\xi+i \eta$ we have

$$
g(v, v)=g(\xi, \xi)-g(\eta, \eta)+2 i g(\xi, \eta) .
$$

Received by the editors July 25, 2008.

2000 Mathematics Subject Classification. Primary 57R25; Secondary 57R20.

(C)2009 American Mathematical Society Reverts to public domain 28 years from publication 
Proof. We show that if $g(v, v) \neq 0$, then $v$ can be deformed to a nowhere zero real vector field. So the Euler characteristic would be zero, according to (1). We decompose $M$ as

$$
M=A_{+} \cup B \cup A_{-},
$$

where $g(\xi, \xi)>g(\eta, \eta)$ on $A_{+}$, the opposite inequality holds on $A_{-}$, and equality holds on $B$. We assume for now that $B$ is not empty. Note that $\xi$ is nowhere zero in $A_{+}$and $\eta$ is nowhere zero in $A_{-}$. Further, since $g(v, v)$ is never zero, we have that $g(\xi, \eta)$ is never zero on $B$. Thus there is an open neighborhood $\Omega$ of $B$ on which $g(\xi, \eta)$ is never zero. We may take $\Omega$ to have a smooth boundary. We have that $\eta$ is never zero in $A_{-} \cup \Omega$ and $\xi$ is never zero in $A_{+} \cup \Omega$. Let $\Omega_{1}$ be an open set chosen so that

$$
B \subset \Omega_{1}, \overline{\Omega_{1}} \subset \Omega
$$

and

$$
\overline{\Omega_{1}} \text { is a neighborhood retract of } \bar{\Omega} \text {. }
$$

The boundary of $\Omega$ has two components, one in $A_{+}$and the other in $A_{-}$. (That is, the boundary of $\Omega$ is the union of two sets, neither of which need be connected.) The same is true for the boundary of $\Omega_{1}$. We will work only with the components in $A_{+}$. Call them $\Sigma$ and $\Sigma_{1}$. Each of these sets separates $M$ into two components. We seek to deform $v$ to a nowhere vanishing real vector field $u$. Set $u=\xi$ on the component of $M-\Sigma$ which does not contain $A_{-}$. The sets $\Sigma$ and $\Sigma_{1}$ bound a region which retracts onto $\Sigma_{1}$. We want to rotate $\xi$ to $\eta$ (or to $-\eta$ ) as the retraction takes $\Sigma$ to $\Sigma_{1}$. Since $g(\xi, \eta) \neq 0$ in this region, this is easily done. Pick a point in this region. The angle $\theta$ between the vectors $\xi$ and $\eta$ satisfies one of the alternatives

$$
0 \leq \theta \leq \pi / 2 \text { or } \pi / 2<\theta \leq \pi,
$$

and whichever alternative is satisfied at that point is also satisfied at all points in the region. Thus as we retract $\Sigma$ to $\Sigma_{1}$, we may rotate $\xi$ to $\eta$, or, respectively to $-\eta$. Finally, define $u=\eta$, respectively $u=-\eta$, on the component of $M-\Sigma_{1}$ which contains $A_{-}$.

If $B$ is empty, the proof is even easier. Now either $g(\xi, \xi)>g(\eta, \eta)$ everywhere and so $\xi$ is a nowhere zero real vector field or the opposite inequality holds and $\eta$ is a nowhere zero real vector field.

Added in Proof. B. Jubin noted that this proof is incomplete. If $\Omega$ is disconnected and $A_{-}$connected, it might not be possible to consistently choose $u= \pm \eta$, so we end up with a line field on $M$ rather than a vector field. Fortunately, the conclusion of Theorem 1 holds also for line fields. This is easily seen by passing to the double covering of $M$ given by the two unit vectors in the line field.

Remark. We have proved the theorem by reducing to (11). This latter result goes back to H. Hopf; an influential modern proof was given by Atiyah 11. Atiyah's proof makes use of the Clifford algebra structure on the bundle of exterior forms. Our theorem can be proved directly, without reducing to (1), by following Atiyah's proof using the corresponding complex Clifford algebra.

There is a stronger version of (11) which expresses the Euler characteristic as the algebraic sum of the indices of the zeros of the vector field. (Indeed this is the result of Hopf.) It would be interesting to generalize this to complex vector fields. 


\section{REFERENCES}

[1] Atiyah, M., Vector fields on manifolds. Arbeitsgemeinschaft für Forschung des Landes Nordrhein-Westfalen, Heft 200, Westdeutscher Verlag, Cologne, 1970, 26 pp. MR0263102 $(41: 7707)$

Department of Mathematics, Rutgers University, Camden, New Jersey 08012

E-mail address: jacobowi@camden.rutgers.edu 